\title{
Reflectance transformation imaging for documenting changes through treatment of Joseon dynasty coins
}

\author{
Jihyun Min'1, Sanghoon Jeong ${ }^{1}$, Kangwoo Park², Yeonghwan Choi ', Daewon Lee ${ }^{3}$, Jaehong Ahn', \\ Donghwan $\mathrm{Har}^{3}$ and Sangdoo Ahn ${ }^{1,5^{*}}$ (D)
}

\begin{abstract}
We present reflectance transformation imaging (RTI) as a documentation tool for visualizing and recording the treatment of coins. RTI - a computational photograph technique that calculates light positions - allows interactive relighting for vision. Virtual light enhances surface details for examining morphological difference. By applying Dome RTI method, stages of conservation treatment were recorded to enhance the overall characteristic features of the relief upon the coin surface, and then detect and identify weathered characters. Patina removal and consolidation were documented along with the original state; a significant difference in the coin's surface was observed using different filters of the RTI viewer. Specular enhancement and normal visualization results were most effective for detecting the change in morphology and reflectivity. Microscopic RTI was applied to visualized minimal changes of characters between treatment stages. Character "常" of coin 1 showed changes in the cleaning of dirt and removal of alumina powder. The character "元" of coin 3, originally covered by a thick patina, revealed clear strokes using virtual relighting through RTI. These documentation images indicate that RTI is a promising tool to support manual recording of conservation stages and, furthermore, allow detection of areas difficult to visualize through the human eye.
\end{abstract}

Keywords: Reflectance transformation imaging, Documentation, Joseon dynasty coinage, Sangpyeong-tongbo, Inscription, Relief, Surface Morphology

\section{Introduction}

Documentation of the treatment process is important for cultural heritage because the record itself is critical information for re-treatment as well as an ethical obligation for the conservator. Preservative proceduresfrom examination by the human eye, recorded notes and sketches, and digital photography to scientific analysisare all valuable for understanding the status of an artifact. Most documentations are supplemented with multiple photograph images and detailed notes. However, no matter how detailed the explanation of the conservator or the

\footnotetext{
*Correspondence: sangdoo@cau.ac.kr

${ }^{1}$ Department of Cultural Properties, Chung-Ang University, Seoul 06974 South Korea

Full list of author information is available at the end of the article
}

photographed images, there are limitations to recording accurate and specific details of the artifact's surface. In this respect, reflectance transformation imaging (RTI) can be an effective tool to aid human vision and record morphological surface and texture.

RTI is an advanced technique using raking light, whereby multiple light sources are distributed over a hemispherical dome and processed using hemispherical harmonics $[1,2]$. It allows interactive re-lighting of the object by saving the data of the light vector on to every pixel. Details hidden because of light and color distortion can also be revealed using RTI viewer filters. Its enhanced vision reveals shallow reliefs and reflectiveness by shading different light angles and excluding color values.

In the literature, studies using RTI have yielded promising results on various ranges of material and type-from

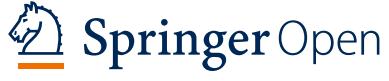

(c) The Author(s) 2021. Open Access This article is licensed under a Creative Commons Attribution 4.0 International License, which permits use, sharing, adaptation, distribution and reproduction in any medium or format, as long as you give appropriate credit to the original author(s) and the source, provide a link to the Creative Commons licence, and indicate if changes were made. The images or other third party material in this article are included in the article's Creative Commons licence, unless indicated otherwise in a credit line to the material. If material is not included in the article's Creative Commons licence and your intended use is not permitted by statutory regulation or exceeds the permitted use, you will need to obtain permission directly from the copyright holder. To view a copy of this licence, visit http://creativecommons.org/licenses/by/4.0/. The Creative Commons Public Domain Dedication waiver (http://creativeco mmons.org/publicdomain/zero/1.0/) applies to the data made available in this article, unless otherwise stated in a credit line to the data. 
near-2D artifacts like inscription tablets to $3 \mathrm{D}$ artifacts like Greek vases [3-5]. RTI is used in diverse environments within the field of archaeology in order to record and reveal manufacture techniques. Dome RTIs are especially used in indoor laboratories and highlighting methods are used in field environments where manual capturing is required [6,7]. Smaller dome types have also been developed for outdoor usage, such as in the studies of Florindi et al. and Porter et al. [8, 9]. In a more recent study by Greaves et al., 3D methods were incorporated into RTI in order to record accurate metric data [10]. RTI has long been recognized as a practical tool for documenting and evaluating conservation treatments by visualizing the change in surface [11-14]. The tool has especially produced outstanding results in understanding hidden information and methods for production when used to examine cultural artifacts with a relatively shallow surface, such as manuscripts, textiles, and paintings [15-19].

Indeed, since its earliest development, RTI has been used to study ancient coins because, first, owing to their small size, the rusty surface with legends and symbols of coins are difficult to visualize; and, second, coins have shallow reliefs that once would have been shiny and reflective, but are now left with corroded surface. We know that RTI has been successfully used for ancient European coins as a documentation method and for interactive contents. In 2005, Mudge et al. reviewed coins from the Hospice of the Grand St. Bernard using a manual system of a polynomial texture map that consisted of a tripod and an extension arm [20]. The authors show RTI to be a superior method compared with traditional numismatic documentation and structured light 3D acquisition for high-quality documentation. Similarly, Kotoula and Kyranoudi found that RTI assists coin identification-Greek and Roman coins, in their case-and further emphasized its advantages for documentation and monitoring of the cleaning process of small-sized artifacts [21]. RTI as an interactive kiosk for storytelling of coin collections also demonstrates its usability in museum education [22, 23]. Indeed, the success of RTI has motivated scientists to improve its capturing ability and combine it with other techniques for evaluation studies. Ciortan et al., for example, presented a practical acquisition and processing pipeline based on free-from RTI, where coins were used to evaluate the result [24]. Hess et al. reviewed RTI with other imaging and analytical techniques to determine the feasibility of digital techniques and their contributions [25].

While the studies discussed above explore in great depth the applications of RTI in documentation, monitoring, and conservation, there are fewer studies that record changes during the stabilization and consolidation process, the last stage of conservation. These stages especially change the surface color and reflectivity of metal artifacts because of the application of a solution that impregnates the surface of the object. In this study, we applied RTI to examine the result of each treatment stage and reveal information hidden under the surface. Through a recording conservation treatment using dome and microscopic RTI systems, the change in visual characteristics and morphological features was maximized. Three Asian coins from the Joseon dynasty were selected for documentation of the conservation process. Many of these ancient coins were used in everyday life as a medium of exchanging goods, leading to natural wear on their surfaces. Then, a significant amount of time under harsh conditions has led them to develop extensive patina, often of an insidious type, causing the metal body to crumble, leaving a pitted surface.

\section{Materials and methods \\ Joseon dynasty coin}

The Joseon dynasty began the minting of coinage to mostly overcome the constraints and inefficiency of the bartering system that commonly used cloth and grain. Paper money was first established in 1401, during the early Joseon dynasty. Only two decades later, the first Joseon-dynasty coinage was minted as Joseon-tongbo ( 朝鮮通寶) in 1423. However, after the Imjin war (15921598) ended with the withdrawal of Japan, the culture and economy suffered collateral damage. One of the measures the government implemented to stabilize and re-build the country was to mint coins. In 1678, Sangpyeong-tongbo (常平通寶) were newly cast, which continued until 1894. These coins remained widespread even in the period of Japanese colonial rule.

In this study, we review the most common of the Sangpyeong-tongbo, namely, Dangiljeon and Dangijeon. These individually represent the value of "one" and "two." All Sangpyeong-tongbo have four identical characters, 常平通寶, in the obverse side. Currency value, province, and the governmental office that produced the coin were embossed on the reverse side, which sometimes displayed a number or an astronomical symbol representing celestial figures, or other characters. These coins are mainly from the late Joseon dynasty period, and were sandcasted using a mother coin for duplication, as described in The Korean Review [26]. The minting technology was derived from China, although the minting technique and components changed throughout the time of the coins' production and usage [27-30]. These kinds of coins are quite common and plenty in Korea because they had been in use until a century ago. Like most coins, they were used in everyday life, as evidenced by the scratches and chipped marks on their surface, as well as 
heavy corroding that has covered the characters, making the inscriptions on coins difficult to interpret.

\section{Conservation treatment}

The conservation process involved cleaning, stabilizing, and consolidation, in that order. A pre-examination of the current status was done to record the structure and the corrosion of the coins (see Table 1). The obverse and reverse images were captured with a Nikon D850 digital camera.

The coins were cleaned after examining their current status. Tertiary patina on the surface was gently removed with a wooden pick and brush. Corrosive substances on the surface were removed with a cotton swab using an abrasive (alumina powder $0.3 \mu$ ) diluted with distilled water. Corrosion and dust were visualized with an optical microscope (see Fig. 1). Coins 1 and 2 showed evidence of cuprite, the first product of copper corrosion, with specks of malachite, which is the secondary patina, a transformation of cuprite. Coin 3 had additional contamination over and underneath the secondary patinamainly soil, and dust with some sand in pits from the sandcasting work. Some areas where malachite was heavily formed were physically removed using dental tools and a surgical knife under an optical microscope following the basic procedure of coin conservation [27]. The chlorides were gently removed to the point where the primary patina was revealed. Residual foreign substances were removed with a soft brush using ethyl alcohol and acetone, and then dried naturally for more than $24 \mathrm{~h}$.

Table 1 Examination of the original state before treatment

\begin{tabular}{|c|c|c|c|c|}
\hline \multirow[t]{2}{*}{ No } & \multirow{2}{*}{$\begin{array}{l}\text { Inscription } \\
\text { Obverse: 常平 } \\
\text { 通寶 } \\
\text { Reverse: 江/二 }\end{array}$} & \multirow{2}{*}{$\begin{array}{l}\text { Original state } \\
\text { A small corrosion of blueish green (malachite) on the front and } \\
\text { overall dark color patina (cuprite) formed on the back }\end{array}$} & \multirow{2}{*}{$\begin{array}{l}\text { Weight size } \\
6.83 \mathrm{~g} \\
30.0 \mathrm{~mm}\end{array}$} & \multirow[t]{2}{*}{ Obverse/reverse } \\
\hline & & & & \\
\hline 2 & $\begin{array}{l}\text { Obverse: 常平 } \\
\text { 通寶 } \\
\text { Reverse: 禁/五/) }\end{array}$ & $\begin{array}{l}\text { Some areas of blueish green corrosion (malachite) on both sides; } \\
\text { some dark color patina areas (cuprite) }\end{array}$ & $\begin{array}{l}4.18 \mathrm{~g} \\
24.9 \mathrm{~mm}\end{array}$ & \\
\hline 3 & $\begin{array}{l}\text { Obverse: 常平 } \\
\text { 通寶 } \\
\text { Reverse: 訓 }\end{array}$ & $\begin{array}{l}\text { Overall corroded in blueish green (malachite) and dark patina } \\
\text { (cuprite); some areas showing blue (azurite); inscribed letters on } \\
\text { the back of the coin are not clearly identified owing to corrosion }\end{array}$ & $\begin{array}{l}4.17 \mathrm{~g} \\
24.6 \mathrm{~mm}\end{array}$ & \\
\hline
\end{tabular}
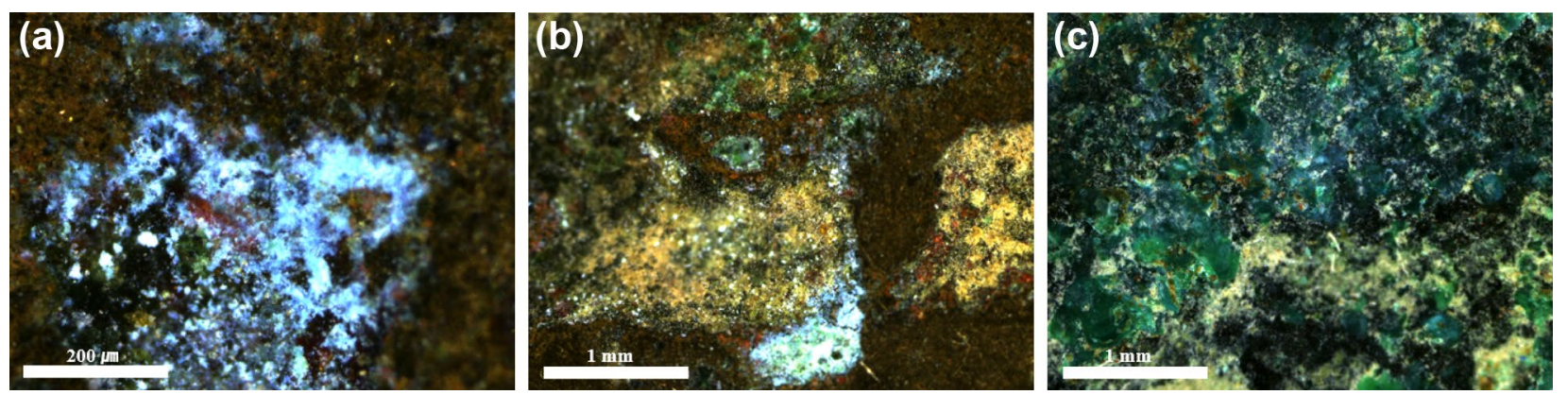

Fig. 1 Microscopic image of corrosion and dust in a Coin 1, b Coin 2, and $\mathbf{c}$ Coin 3 
Consolidation was done to stabilize and protect the metal body and surface from further chemical reaction. To control the further activation of chloride, the coins were immersed in 3\% benzotriazole dissolved in ethanol at room temperature. After impregnation, the surface was wiped several times and naturally dried to ensure no residue remained on the surface. The material was reinforced through treatment using Incralac $^{\circledR}(85 \%$ of acetone: toluene 1:5, 14.6\% of Paraloid ${ }^{\circledR}$ B-72, and 0.4\% benzotriazole), which blocks re-corrosion factors, moisture, and contaminated air from the metal surface. The coins were again immersed into the Incralac ${ }^{\circledR}$ for more than $6 \mathrm{~h}$ to penetrate the coin to form a protective film on the metal. The film was left to fully dry under room temperature for more than $12 \mathrm{~h}$. The coins were finally wiped with Kimwipes ${ }^{\circledR}$ to prevent excessive chemicals from remaining on the surface, which can create an unwanted glossy surface.

\section{Reflectance transformation imaging Dome RTI}

The RTI images were captured using a dome-type RTI system (Fig. 2). The RTI has a rail system for height adjustment and mobility. The LED circuits were connected in parallel to lessen structural sensitivity. Each group of dataset had 45 images, captured with a Nikon D850 DSLR camera with an AF-S Micro Nikon $105 \mathrm{~mm}$ 1:2:8G ED lens. We attained $8256 \times 5504$ pixels per image with a CMOS sensor type camera. As shown in Fig. 1, the inner wall of the dome had a $50 \mathrm{~cm}$ radius, and 45 LEDs were attached in a geodesic order to obtain equal lighting angle and distance for every image. The angle to the surface of the coin was L1, 5 to $10^{\circ}$; L2, 25 to 40 degrees; L3, 50 to $65^{\circ}$; and L4, 70 to $80^{\circ}$. The 45 set of LEDs had a color temperature value of $4000 \mathrm{k}$, which is a neutral white color considered to be the midpoint between warm white and cool white. These LED were selected as a light source for correct color reproduction compared with other studies that used various color temperature types of LEDs. A black glossy ball was used to register each light position to create a light position (lp) file for this array. The acquisition time for one sequence was two minutes, which was started by pushing a button from the controller. The images were captured in a RAW file to save the full metadata of the coin. This information was recorded together with each RTI dataset and can be critical information for later data analysis and also serves as a digital replica [12]. Using Adobe Bridge ${ }^{\circledR}$ converter, the images were exported as.jpeg files. The captured images were processed with the lp.file using the RTI Builder software, provided as an open source software by Cultural Heritage Imaging [31]. The final file was processed by selecting a polynomial texture map (.ptm) format or a hemispherical harmonic (.rti) format $[1,2]$. We chose the latter format because it guaranteed better reproduction [32]. The result of the rendering process can be visualized through the RTI viewer. The image can be virtually re-lighted in different directions in real time and its color, texture, and reflective property can be adjusted through filters. The approximate spatial resolution for pixel per $\mathrm{mm}$ was 5.7 .

\section{Microscopic RTI}

The microscopic RTI was performed using an optical microscope and a single LED, as shown in Fig. 3. Zeiss Smart Zoom 5 was selected to acquire microscopic images. The significantly wide working space $(16 \mathrm{~cm} \times 16 \mathrm{~cm})$ allowed for the shedding of light in different directions around the characters on the coin's surface. We attained $1600 \times 1200$ pixels per image with a CMOS sensor type camera. A highlight method was chosen for RTI image capturing using a $2 \mathrm{~mm}$ (r) black shiny ball for the acquisition of each light position. The ball was placed near the area of interest, but not above or too close because of the disturbance of shadows formed by the ball. While other methods such as built-in devices do exist, the highlight method which is done by shedding light manually was practical, given the time required for capturing and the sufficient space around the stage to shed light in different angles compared with developing a new device [15]. An average of 40 images were captured
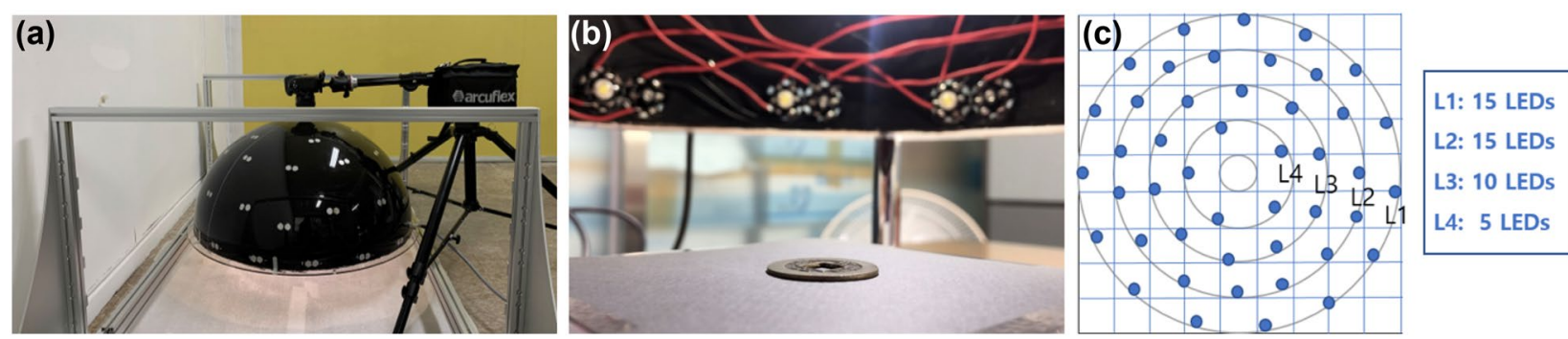

Fig. 2 a Dome type RTI system and camera stationing; $\mathbf{b}$ interior image of RTI shooting system; c LED distribution 


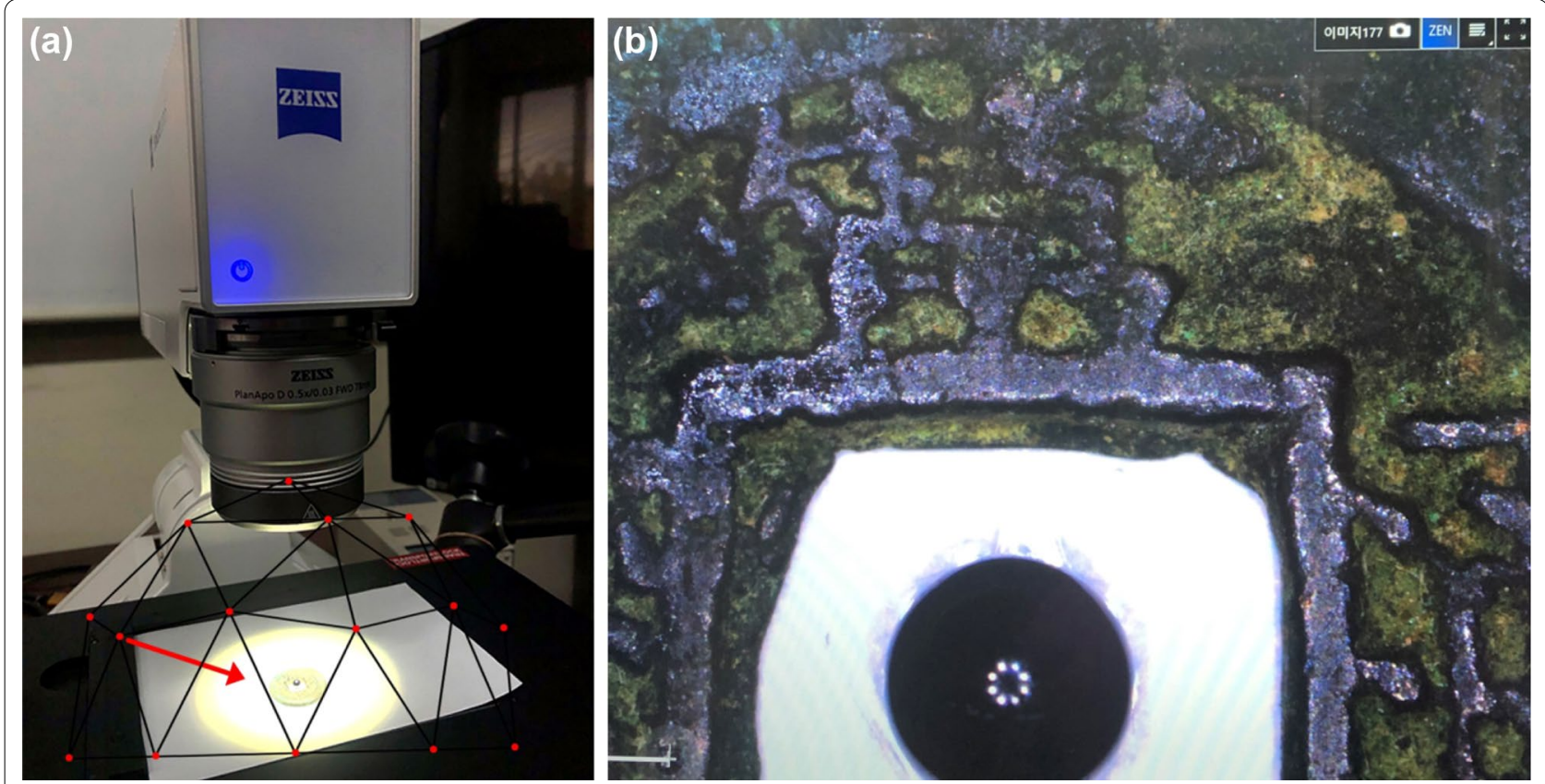

Fig. 3 a Microscopic RTl; b location to be captured under the microscope examined 20 times the actual size

for each set. The acquisition time was approximately three minutes to manually move the LED light from one position to another from the desired distance and angle. The captured images were equally processed through the RTI Builder, and then visualized through the RTI Viewer. The approximate spatial resolution for pixel per $\mathrm{mm}$ was 3.8 .

\section{Results and discussion}

\section{Documentation of treatment stage using dome RTI}

Virtual relighting and filters were applied to visualize the status of each coin. Yellowish brown dust that was piled near and between the characters were clearly observed in all three coins. An area covered with dirt in Coin 1 had a different surface specularity, as seen in Fig. 4a. During cleaning, the dirt was removed to reveal a hole in the surface, which was observed as a dark empty area in Fig. 4b. The surface of the pit revealed no sign of corrosive substances; therefore, the pit may have been a minor flaw that occurred during the casting of the coin. The wide rim surrounding the characters showed much deeper scratches after cleaning.

The RTI image effectively shows the dynamic change of surface morphology before and after cleaning stages in Coin 3 (Fig. 5). The cleaning process showed a visible difference in normal variation when we compared the changes in the RGB color components, where RGB corresponded to the $\mathrm{X}, \mathrm{Y}$, and $\mathrm{Z}$ coordinates, respectively, of the surface normal. The original state that had a relatively even blue color changed to a mixture of red, green, and
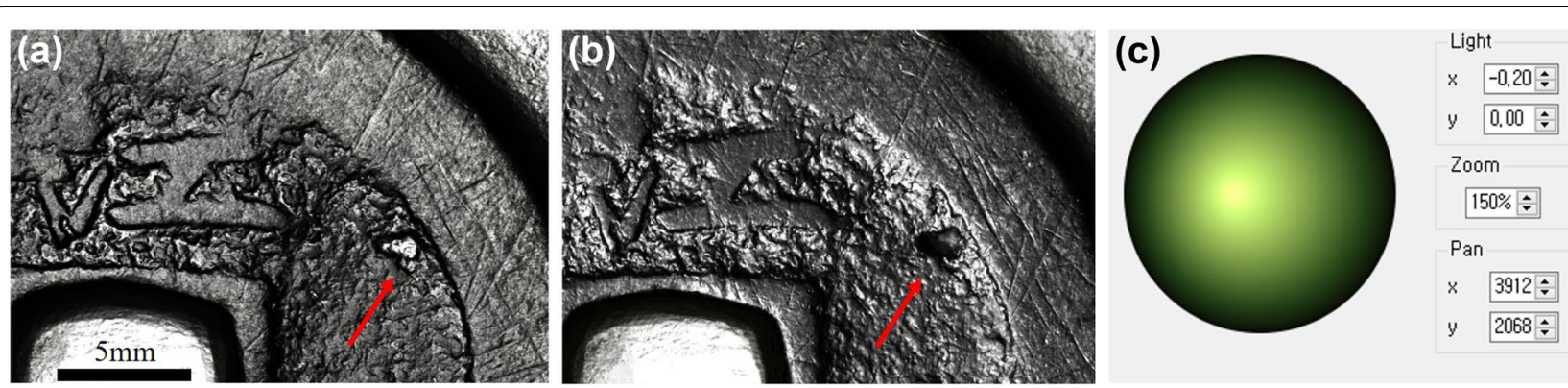

Fig. 4 Visualization of dirt removed from hole on the reverse side of Coin 1 acquired by dome RTI specular enhancement: $\mathbf{a}$ original state, $\mathbf{b}$ after cleaning, $\mathbf{c}$ light coordinate (where $x$ is -0.20 and $y$ is 0.00 ) 

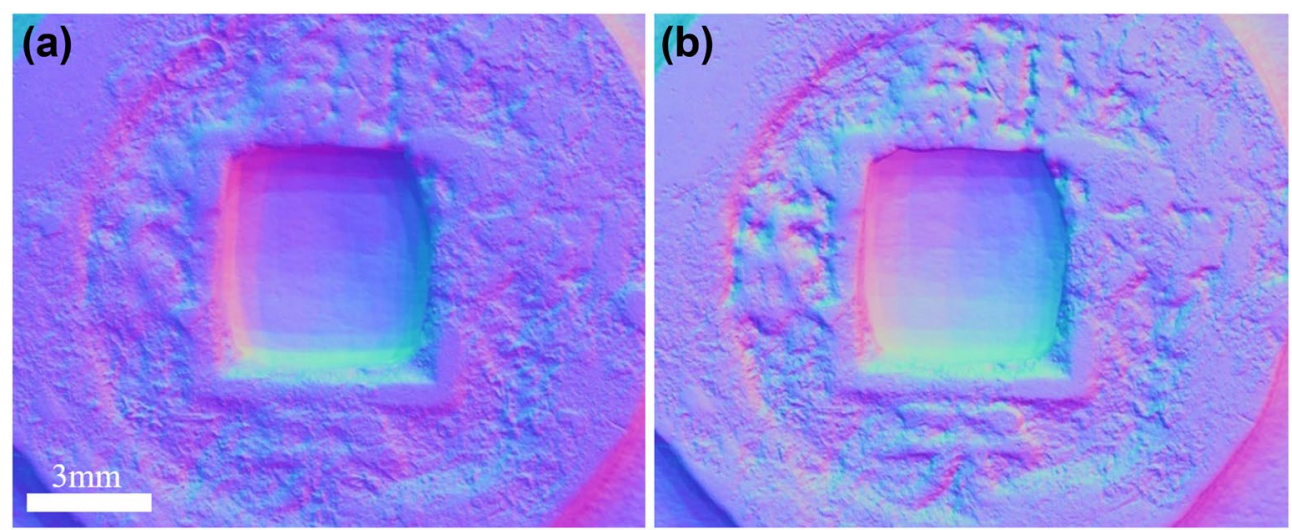

Fig. 5 Change of surface morphology in the reverse side of Coin 3 visualized by normal vector map image acquired by dome RTI: $\mathbf{a}$ original state, $\mathbf{b}$ after cleaning state showing different normal variation

blue, especially near the characters, indicating changes in surface morphology.

The sandcasting methods left an uneven surface with shallow pits made by sand grain, where abrasives for cleaning resided after polishing the coins. These areas were checked under the microscope and visualized using RTI diffuse gain filters (Fig. 6). Diffuse gain filters increase the representation of the height depth, that is, the points at which the height of an area quickly changes on the surface. In Fig. 6b, dark lines visualize the difference in height depth around the areas where white alumina powder is pitted. This area was then analyzed with a Hitachi S-3400N scanning electron microscope-energy dispersive spectroscopy (20 keV, magnification of $300 \times$, working distance of $10 \mathrm{~mm}$ ) to confirm the white residue powder as alumina. As shown in Fig. 6d, alumina (Al) was detected in that area ( $\mathrm{Al} \mathrm{2.55 \% ,} \mathrm{Si} 0.42 \%$, Fe 3.30\%, $\mathrm{Cu}$ 87.67\%, Zn 0.26\%, Sn 1.47\%, Pb 4.17\%). Meanwhile, the average composition of the other positions in Coin 1 (Si $0.81 \%$, Fe $2.31 \%$, Cu $62.25 \%$, Zn $8.57 \%$, Sn $8.50 \%$, Pb $20.90 \%$ ) was observed to be similar to the ranges of the Joseon Dynasty coins $[29,30]$. The after-cleaning stage was recorded using RTI prior to the stabilization stage to ensure no residue was left behind, and the abrasive was removed before moving on to the next stage.

The final stage after consolidation was also captured using RTI to record all three stages (Table 2). The images in Table 2 were all acquired under the same RTI light position. Specular enhancement images and normal map images were selected to record the changes of the coin's surface because these filters best visualize the change in surface reflectivity and morphology.

Figure 7 shows a better visualization of the changes in surface specularity. Comparing the original state and the after-cleaning state of Coin 2 shows that the surface became glossier through cleaning of dust and patina. In comparison, in the after-consolidation state, the coin appears dark and blurry. This is because specularity diminishes because of the coated layer of Incralac ${ }^{\circledR}$ as a result of stabilization and consolidation.

\section{Detailed visualiztion using microscopic RTI}

Detailed images were attained using microscopic RTI with a magnification of 20 and 30 for Coin 1 and Coin 3 . The letter “常” in Coin 1 was taken to examine the process of excluding corrosive substances near the upper area of the inscription. Each stage of treatment was visualized with different filters (Fig. 8). The most substantial change was observed when we compared the specular enhancement and normal map images. Specular enhancement showed the change in surface morphology, where deep scratches under the dust were revealed. The normal map images also showed the same features, where normal vectors were transformed into the RGB color component. However, deep scratches were more apparent in specular enhancement images because of the filter effect used reflectivity as a tool for vision. Also, the removal of foreign matters can be visualized through the normal map. Yellow dust packed in the pits of the sand-casted surface were thoroughly removed from the surface, and this change can be seen in a comparison with the normal map image. The after-consolidation images revealed the result of coating of solution, where shallow scratches and dents from everyday usage were filled, as shown in Fig. 8c. Coin 1 showed a significant difference in color between the microscopic image and the RTI result. The microscopic image was captured using stationed light attached to the microscope. All three results of the original microscopic images gave poor results in color and detail. The image's true color was not attained, because of the microscope's 

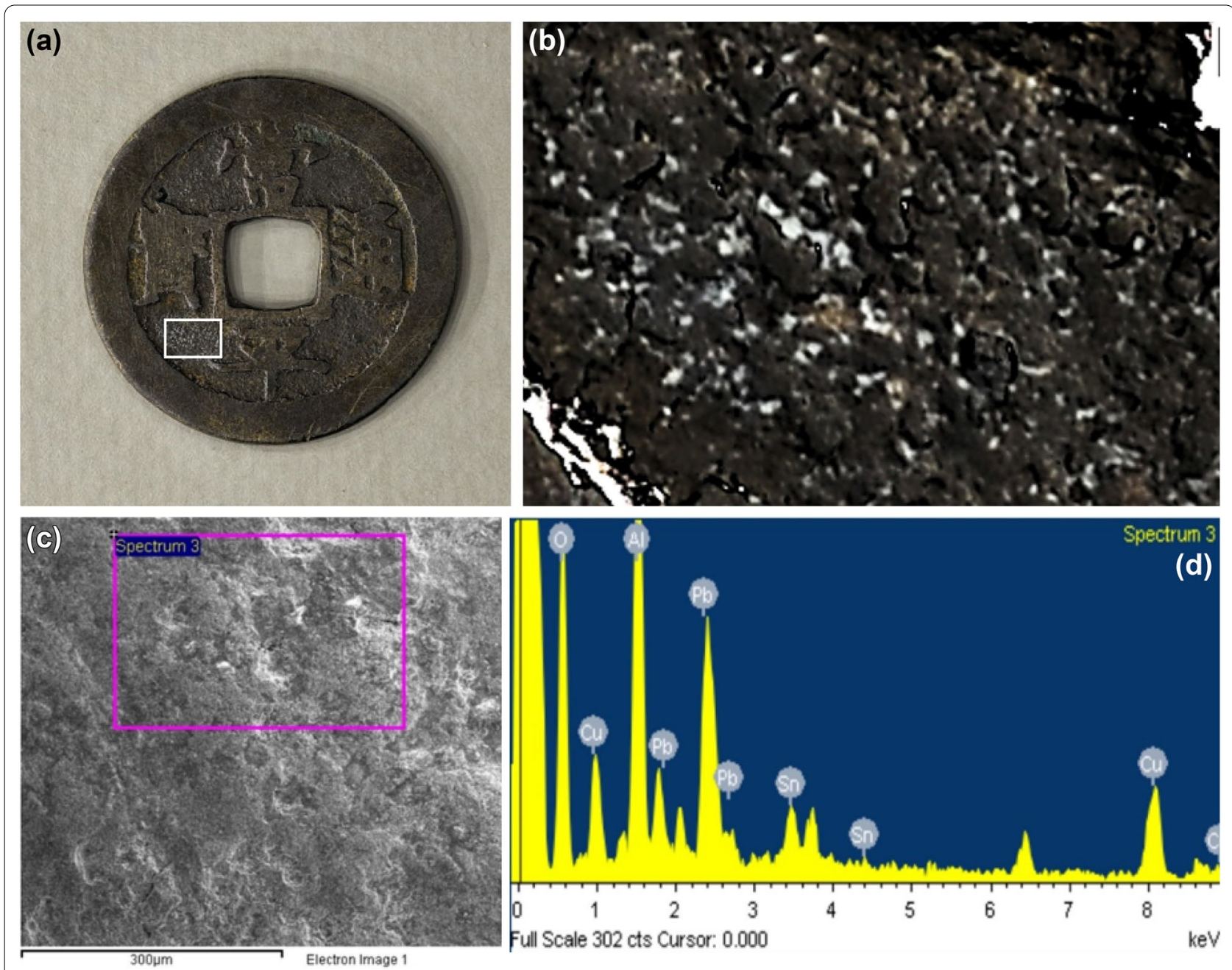

Fig. $\mathbf{6}$ a Location of abrasive residue and $\mathbf{b}$ magnification of white box area $\mathbf{a}$ using diffuse gain filter; $\mathbf{c}$ analysis of abrasive residue using scanning electron microscope-energy dispersive spectroscopy and $\mathbf{d}$ detected peaks of selected region where alumina spectrum (Al) is shown

light source, whereas in the RTI-Normal Unsharp images, realistic color was attained. In addition, the original microscopic image lacked surface detail because of the reflective features of the surface, whereas the RTISpecular Enhancement and RTI-Normal Map revealed scratches and sand-casting marks upon the surface.

The character “元” in Coin 3 was revealed through RTI observation. As shown in Fig. 9a, the microscopic image scarcely revealed the character. Similar to the case above in Coin 1, original microscopic images of Coin 3 yielded less-effective results because of the reflection and gloss of the coin's surface especially after cleaning and consolidation. However, through the application of RTI, a hint of the character's shape was revealed. The cleaning process of the heavily covered patina was aided through the RTI result. After cleaning, areas of corrosive substances that were visualized in the normal vector map as red and green were removed to reveal the original surface, which shows as blue. The blue color indicates the $z$ axis $(0,0,1)$, corresponding to a vector facing directly out from the surface. The result of the removal of cupric rust can also be seen in the specular enhancement image. Specular enhancement image (b) also revealed a chipped area at the lower left stroke, which was not detected in any of the original microscopic images.

In Fig. 10, horizontal and vertical strokes of the character “元” in Coin 3 were dramatically visualized with specular enhancement filter by shading different directions of light. Compared to images of Fig. 9 which were captured under a perpendicular light angle, images of Fig. 10 gave better visualization by shading different light angles. The strokes were best visualized when shading light in a vertical angle to the stroke of interest. 
Table 2 Normal vector map and specular enhancement on the obverse and reverse sides of coins acquired by dome RTI: (a) original photograph image; (b), (d), (f) specular enhancement filter; (c), (e), (g) normal vector map; O and R stand for obverse and reverse, respectively

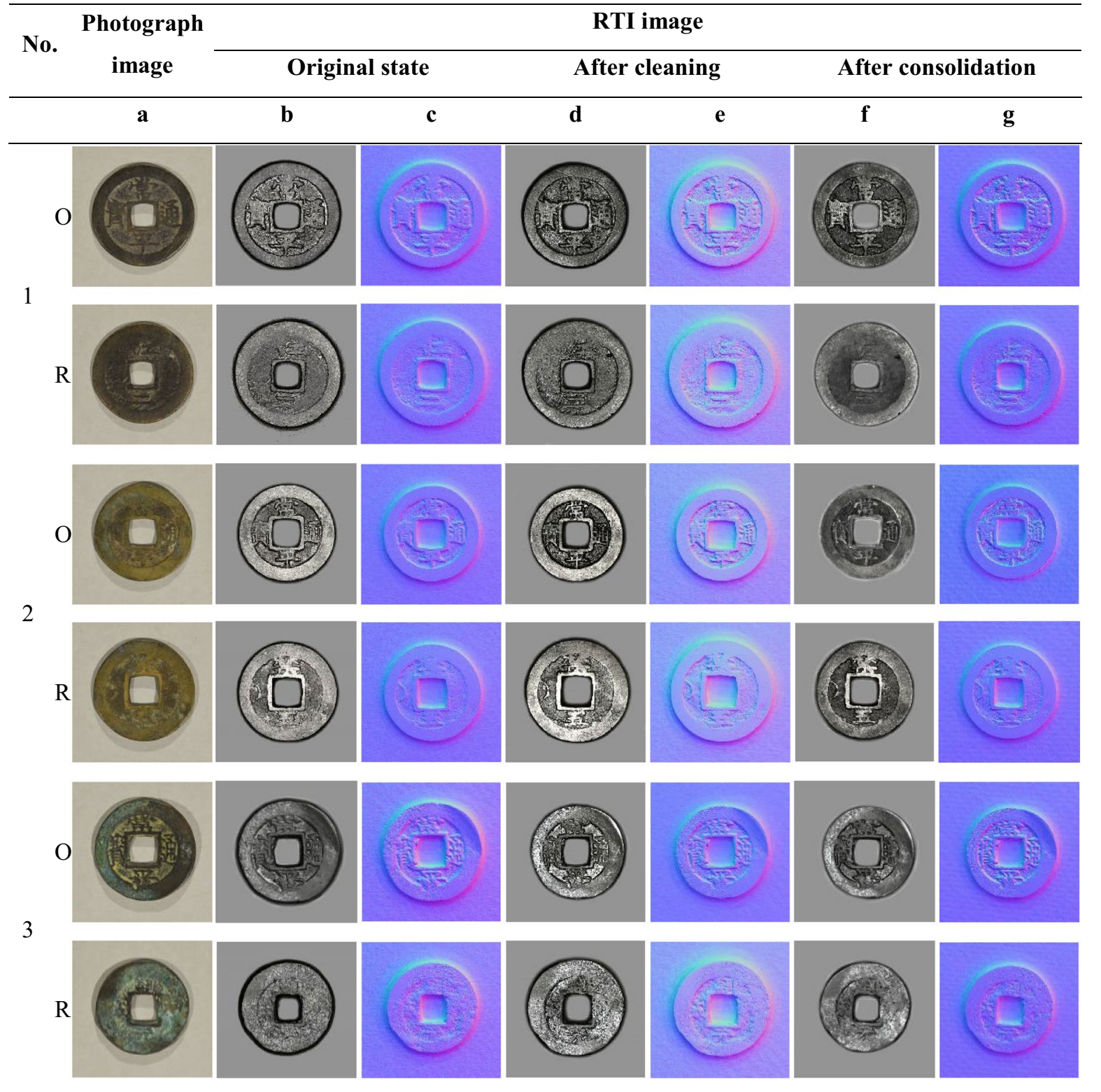

\section{Conclusions}

Through this study, we find that the most advantageous features of RTI are in visualizing the changes in the process of conservation. The images themselves can be used as an interactive tool to evaluate the current status and record the process and result of conservation. In addition, the two types of RTI methods herein yielded detailed information, with a marked reduction with regard to light control and stage preparation than original photography. In particular, the characters and scratches from everyday usage hidden under the dust and patina were better visualized through RTI filters. In some areas, RTI images helped detect the strokes of the characters in the initial stage of conservation. RTI imaging filters such as specular enhancement and normal map filters helped detect characters covered with patina. Diffuse gain filters corrected areas with a build-up of alumina residue.

Overall, RTI shows promising results in recording changes in both broad and detailed surfaces. Through Dome RTI, the overall change in surface reflectivity, 

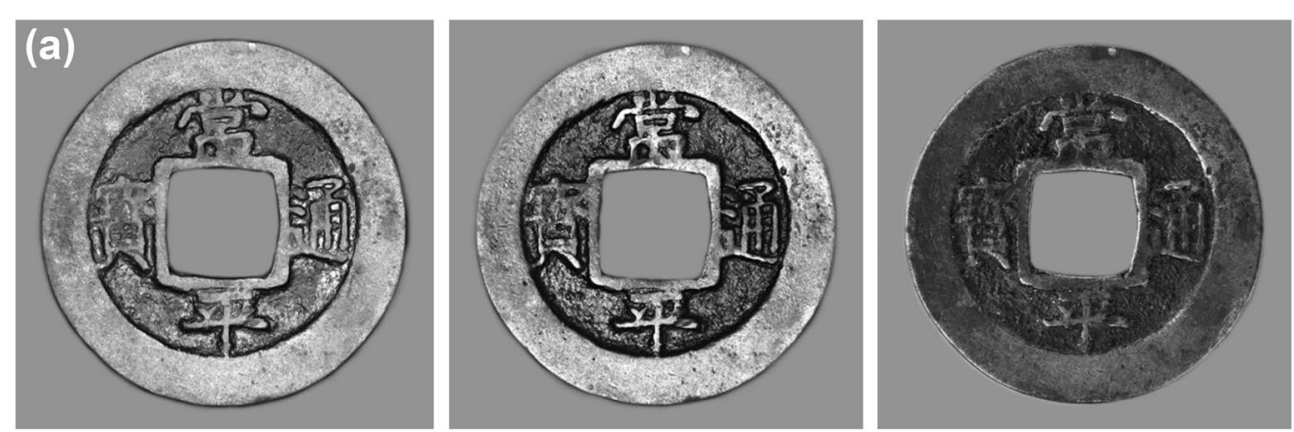

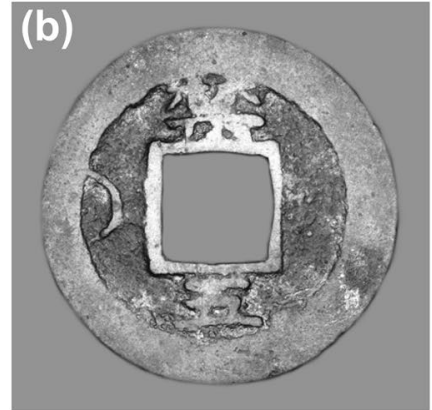

Original State

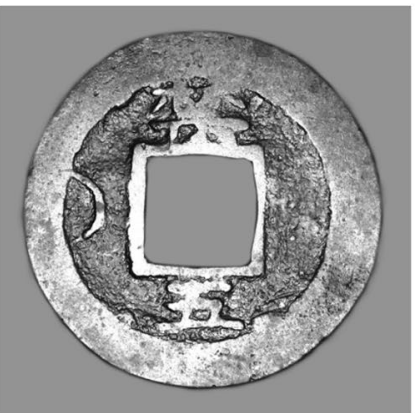

After Cleaning

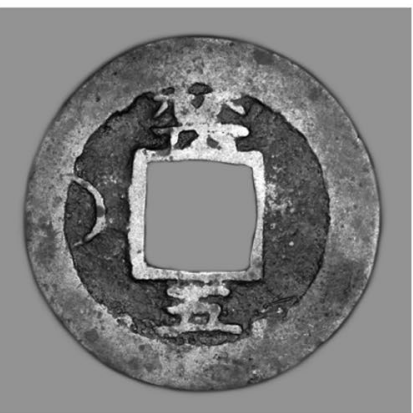

After Consolidation

Fig. 7 Different features of reflectiveness of Coin 2 after the process of cleaning and consolidation (magnified images from Table 2): a obverse side and $\mathbf{b}$ reverse side
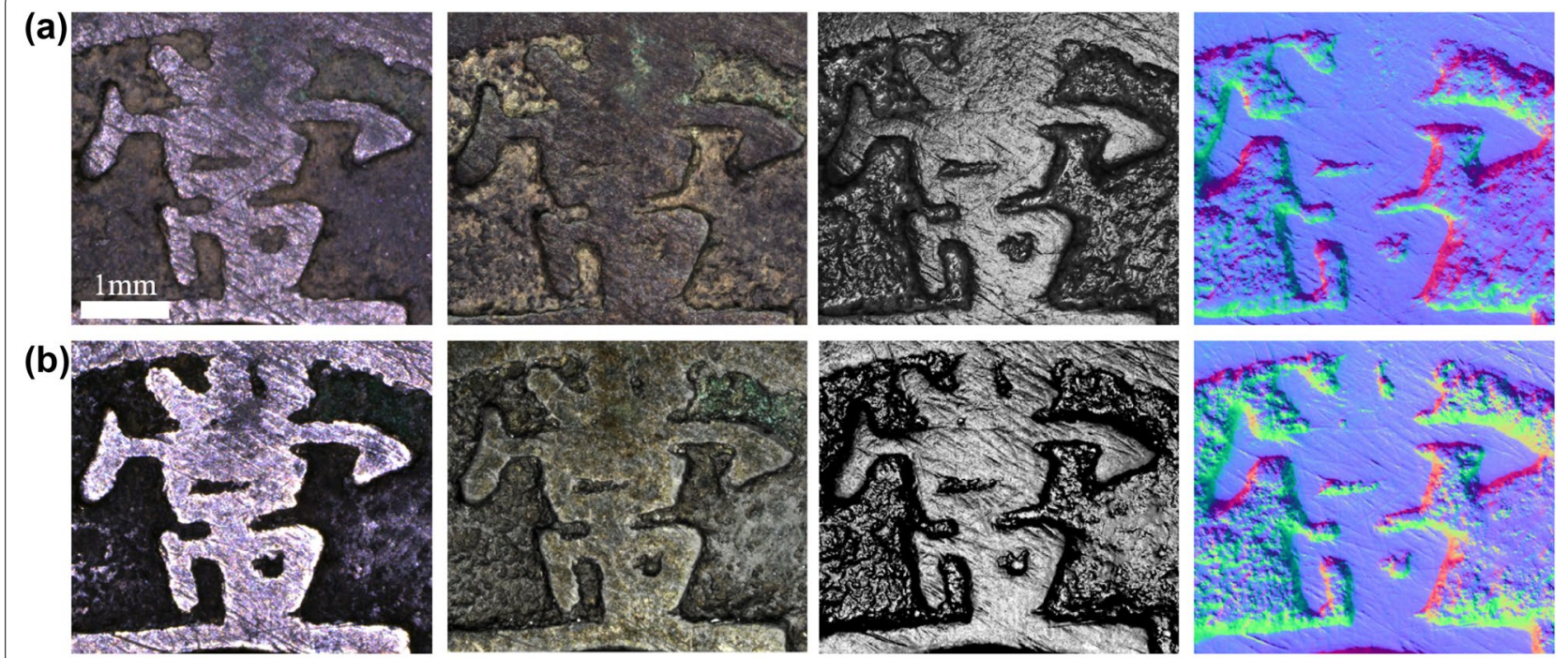

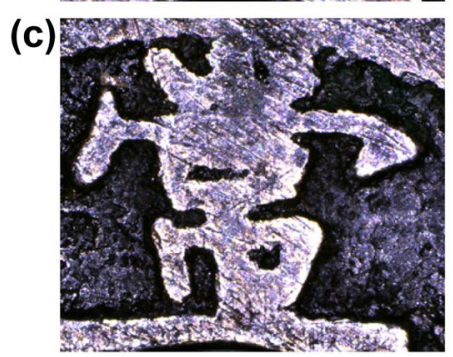

Original Image

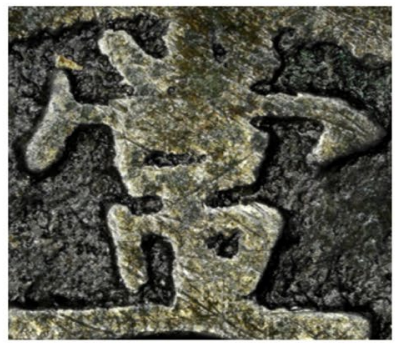

RTI-Normal Unsharp

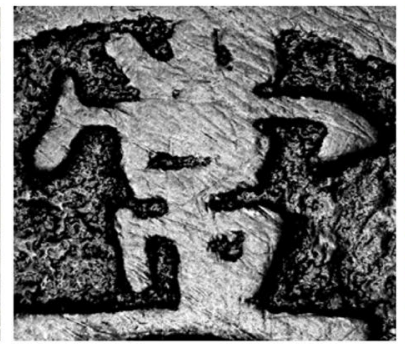

RTI-Specular Enhancement

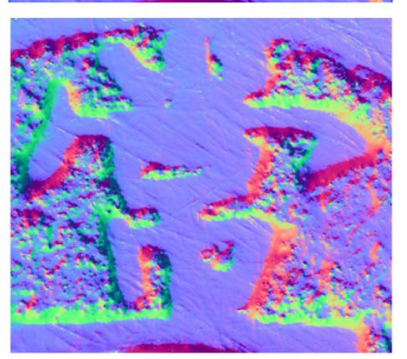

RTI-Normal Map

Fig. 8 Microscopic RTI image of character "常" of Coin 1 observed with various filters: a original state, $\mathbf{b}$ after cleaning, and $\mathbf{c}$ after consolidation 


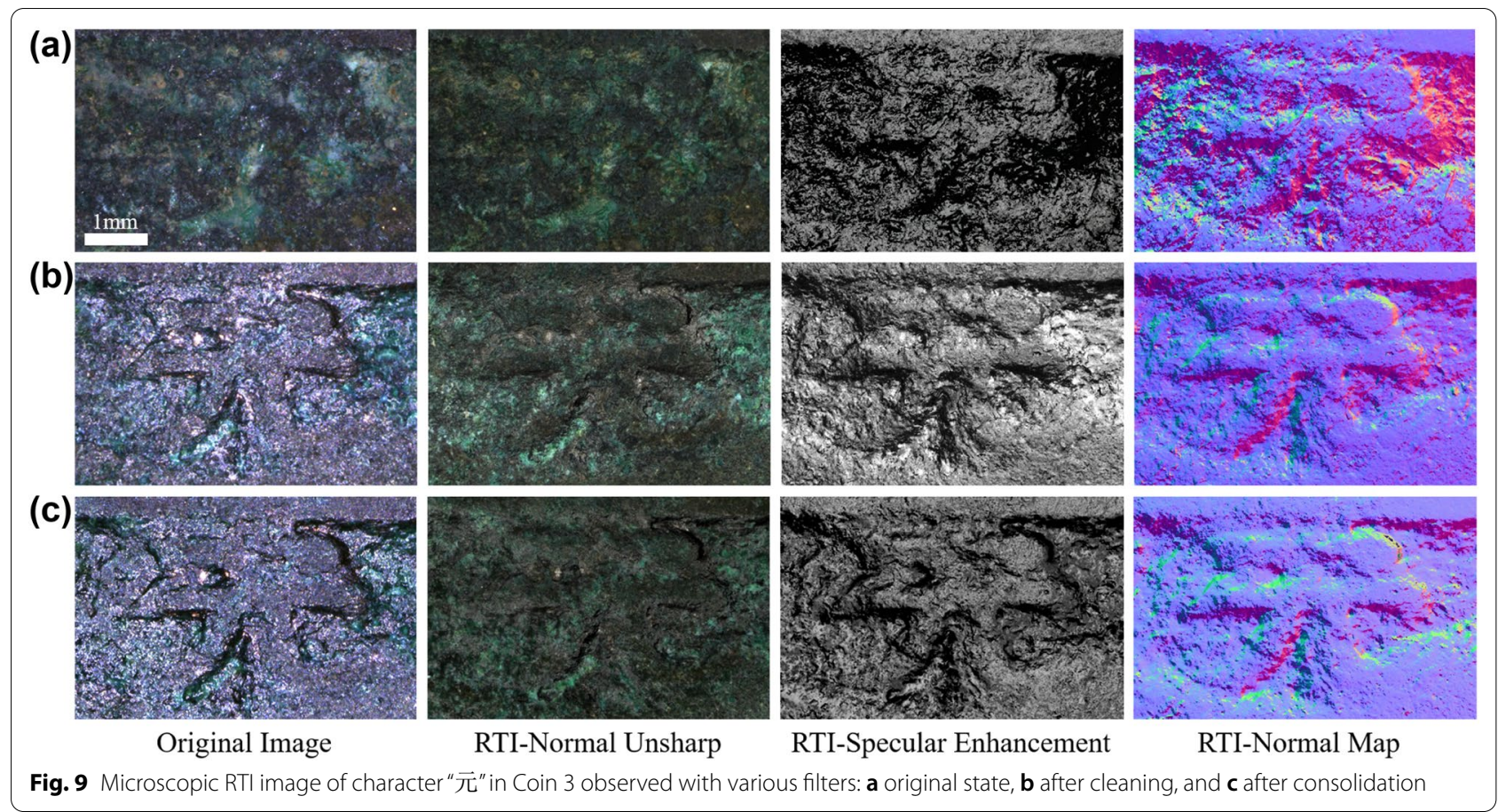

as well as features such as scratches and chipped areas resulting from everyday usage were revealed. Microscopic RTI captured changes in characters where morphological changes on the surface were recorded after cleaning and coating, whereupon scratches were revealed and then filled up with consolidation solution. Furthermore, better character recognition was possible by virtually relighting the characters of the coin. In particular, the after-consolidation images can offer insights to conservators on minimal surface change, such as reflectivity and applied state of coating.

Finally, the documented results reinforce the myriad applications of RTI. For example, RTI images can be a practical option for rapidly corroding artifacts excavated from harsh environments because they can allow conservators to sensitively and thoroughly detect changes in the artifacts' surface using virtual re-lighting and RTI filters. Moreover, RTI specular enhancement
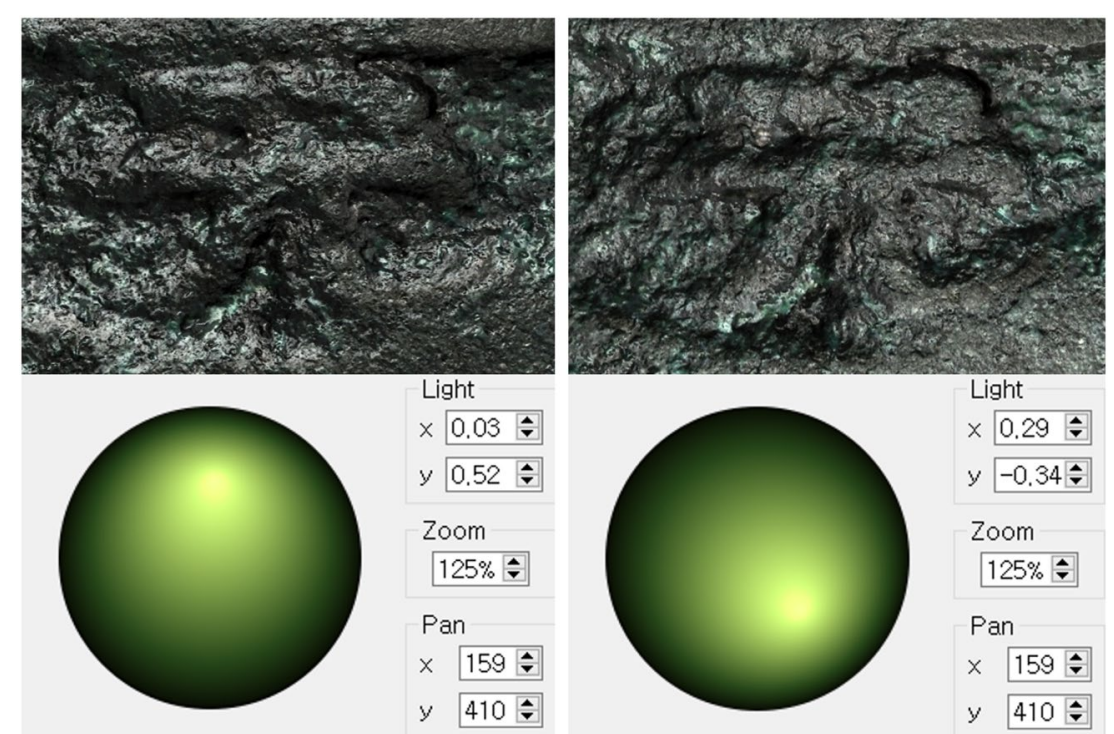

Fig. 10 Visualization of the strokes of the character “元" in Coin 3 by different angles of light. The highlighted spot on the sphere marks the direction of the light 
images can serve as digital rubbing images. This can be a possible alternative method to traditional rubbing, as the latter may cause harm to the artifact in several ways, such as contaminating the surface with ink stains or blunting details on the weakened surface.

\section{Acknowledgements}

We thank Shinyoung Science and Carl Zeiss for supporting the experiment by renting us Zeiss Smart Zoom 5.

\begin{abstract}
Authors' contributions
Conceptualization: JM and SA; methodology: JM, SJ, KP, and JA; software: JM, SJ and DL; validation: JA and SA; formal analysis: JM and YC; investigation: JM and SA; resources: YC, DH, JA and SA; data curation: JM and SJ; writing-original draft preparation: JM; writing — review and editing: DL and SA; visualization: JM, SJ and KP; supervision: DH and SA; project administration: JA and SA; funding acquisition: SA. All authors have agreed to the published version of the manuscript. All authors read and approved the final manuscript.
\end{abstract}

\section{Funding}

This research received no external funding.

\section{Availability of data and materials}

The datasets used and/or analyzed during the current study are available from the corresponding author on reasonable request.

\section{Declarations}

\section{Competing interests}

The authors declare no conflict of interest.

\section{Author details}

1 Department of Cultural Properties, Chung-Ang University, Seoul 06974, South Korea. ${ }^{2}$ Graduate School of Advanced Imaging Science, Chung-Ang University, Seoul 06974, South Korea. ${ }^{3}$ College of Art and Technology Chung-Ang University, Anseong 17546, South Korea. ${ }^{4}$ Graduate School of Culture Technology, KAIST, Daejeon 34141, South Korea. ${ }^{5}$ Department of Chemistry, Chung-Ang University, Seoul 06974, South Korea.

Received: 17 March 2021 Accepted: 26 August 2021

Published online: 08 September 2021

\section{References}

1. Malzbender T, Gelb D, Wolters H. Polynomial texture maps. In: Proc. ACM SIGGRAPH 28; 2001. p. 519-28.

2. Gautron P, Krivanek J, Pattanaik S, Bouatouch K. A novel hemispherical basis for accurate and efficient rendering. In: Eurographics symposium on rendering; 2004. p. 321-30. https://doi.org/10.2312/EGWR/EGSR04/ 321-330.

3. Artal-Isbrand P, Klausmeyer P. Evaluation of the relief line and the contour line on Greek red-figure vases using reflectance transformation imaging and three-dimensional laser scanning confocal microscopy. Stud Conserv. 2013;4:338-59. https://doi.org/10.1179/2047058412Y.0000000077.

4. Piquette KE. Reflectance transformation imaging (RTI) and ancient Egyptian material culture. Damqatum CEHAO NewsI. 2011;7:16-20.

5. MacDonald L, Bennett T, Ramsey C, Crowther C. New RTI technology for palæography. In: Proceedings of EVA London; 2019. https://doi.org/10. 14236/ewic/EVA2019.34.

6. Mytum H, Peterson JR. The application of reflectance transformation imaging (RTI) in historical archaeology. Hist Archaeol. 2018;52:489-503. https://doi.org/10.1007/s41636-018-0107-x.

7. Kotoula E, Robinson DW, Bedford C. Interactive relighting, digital image enhancement and inclusive diagrammatic representations for the analysis of rock art superimposition: the main Pleito cave (CA, USA). J Archaeol Sci. 2018;93:26-41. https://doi.org/10.1016/j.jas.2018.02.012.
8. Florindi S, Revedin A, Aranguren B, Palleschi V. Application of reflectance transformation imaging to experimental archaeology studies. Heritage. 2020;3(4):1279-84. https://doi.org/10.3390/heritage3040070.

9. Porter ST, Huber N, Hoyer C, Floss H. Portable and low-cost solutions to the imaging of Paleolithic art objects: a comparison of photogrammetry and reflectance transformation imaging. J Archaeol Sci. 2016;10:859-63. https://doi.org/10.1016/j.jasrep.2016.07.013.

10. Greaves AM, Duffy S, Peterson JR, Tekoğlu R, Hirt A. Carved in stone: field trials of virtual reflectance transformation imaging ( $\mathrm{V}-\mathrm{RTI})$ in classical Telmessos (Fethiye). J Field Archaeol. 2020;45(7):542-55. https://doi.org/ 10.1080/00934690.2020.1804135.

11. Earl G, Martinez K, Malzbender T. Archaeological applications of polynomial texture mapping: analysis, conservation and representation. J Archaeol Sci. 2010;37:2040-50. https://doi.org/10.1016/j.jas.2010.03.009.

12. Tamayo $M$, Andrés $V$, Pons $O$. Applications of reflectance transformation imaging for documentation and surface analysis in conservation. Int J Conserv Sci. 2013;4:535-48.

13. Boute R, Hupkes M, Kollaard N, Wouda S, Seymour K, Wolde L. Revisiting reflectance transformation imaging (RTI): a tool for monitoring and evaluating conservation treatments. In: IOP conference series: materials science and engineering, vol. 364; 2018. p. 012060. https://doi.org/10. 1088/1757-899X/364/1/012060.

14. Ono S, Matsuda Y, Mizuochi T. Development of a multispectral RTI system to evaluate varnish cleaning. In: ICOM-CC 18th triennial conference. 2017.

15. Goldman Y, Linn R, Shamir O, Weinstein-Evron M. Micro-RTI as a novel technology for the investigation and documentation of archaeological textiles. J Archaeol Sci Rep. 2018;19:1-10. https://doi.org/10.1016/j.jasrep. 2018.02.013.

16. Hanneken TR. New technology for imaging unreadable manuscripts and other artifacts: integrated spectral reflectance transformation. In: Ancient worlds in digital culture. Leiden: Digital Biblical Studies; 2016. p. 180-195. https://doi.org/10.1163/9789004325234_010.

17. Min J, Yoo E, Choi H, Ahn S, Ahn J, Ahn S. Interpretation through digital imaging: reflectance transformation imaging (RTI) as a tool for understanding paintings. Int J Contents. 2020;16(2):41-50. https://doi.org/10. 5392/IJoC.2020.16.2.041.

18. Min J, Ahn J, Ahn S, Choi H, Ahn S. Digital imaging methods for painting analysis: the application of RTI and 3D scanning to the study of brushstrokes and paintings. Multimed Tools Appl. 2020;79:25427-39. https:// doi.org/10.1007/s11042-020-09263-0.

19. Hughes-Hallet M, Young C, Messier P. A review of RTI and an investigation into the applicability of micro-RTI as a tool for the documentation and conservation of modern and contemporary paintings. J Am Inst Conserv. 2020. https://doi.org/10.1080/01971360.2019.1700724.

20. Mudge M, Voutaz JP, Schroer C, Lum M. Reflection transformation imaging and virtual representations of coins from the Hospice of the Grand St. Bernard. VAST. 2005;6:29-40.

21. Kotoula E, Kyranoudi M. Study of ancient Greek and Roman coins using reflectance transformation imaging. E-conserv Mag. 2013;25:74-88.

22. Palma G, Badassari M, Chiara Favilla M, Scopigno R. Storytelling of a coin collection by means of RTI images: the case of the Simoneschi collection in Palazzo Blu. In: Museums and the web, Florence. 2014.

23. Palma G, Siotto E, Proesmans M, Baldassari M, Baracchini C, Batino S, Scopigno R. Telling the story of ancient coins by means of interactive RTI images visualization. In: Archaeology in the digital era; 2014. p. 177.

24. Ciortan IM, Pinuss R, Marchioro G, Daffara C, Giachetti A, Gobbetti E. A practical reflectance transformation imaging pipeline for surface characterization in cultural heritage. Proc GCH. 2016;3:127-36. https://doi.org/ 10.2312/gch.20161396.

25. Hess M, MacDonald LW, Valach J. Application of multi-modal 2D and 3D imaging and analytical techniques to document and examine coins on the example of two Roman silver denarii. Herit Sci. 2018. https://doi.org/ 10.1186/s40494-018-0169-2.

26. Hulbert HB. The Korea review, a Korean mint. 1905; 5(3):87-9.

27. Jeon $\mathrm{H}$, Kang H, Ahn J. Conservation and analysis of Goryeo-era coins in collection of the National Museum of Korea -Donggukjungbo, Dongguktongbo, Haedongtongbo, Haedongjungbo, Samhanjungbo-. Conserv Sci Mus. 2008;9:17-29. https://doi.org/10.22790/conservation.2008.9.0017. 
28. Kang HT, Kim GH, Huh WY, Hirao Y. Raw material and provenance of Chosen-Tongbo (I). J Conserv Sci. 2004;16:15-20.

29. Yun Y, Cho N, Jeong Y, Lim I. Study on the casting technology and restoration of "Sangpyong Tongbo." Korean J Cult Herit Stud. 2014;47:224-43. https://doi.org/10.22755/kjchs.2014.47.4.224.

30. Jang $\mathrm{S}, \mathrm{Cho} \mathrm{H}$, Kang H. Study on the materials characteristics of Sangpyeongtongbo coins in Joseon dynasty using chemical compositions and microstructures. J Conserv Sci. 2015;31:319-30.

31. Cultural Heritage Imaging. http://culturalheritageimaging.org. Accessed 11 Dec 2020
32. Mudge M, Malzbender T, Chalmers A, Scopigno R, Davis J, Wang O, Barbosa J. Image-based empirical information acquisition, scientific reliability, and long-term digital preservation for the natural sciences and cultural heritage. Eurographics (Tutorials). 2008. https://doi.org/10.2312/ egt.20081050.

\section{Publisher's Note}

Springer Nature remains neutral with regard to jurisdictional claims in published maps and institutional affiliations.

\section{Submit your manuscript to a SpringerOpen ${ }^{\odot}$ journal and benefit from:}

- Convenient online submission

- Rigorous peer review

- Open access: articles freely available online

- High visibility within the field

- Retaining the copyright to your article

Submit your next manuscript at $\boldsymbol{\nabla}$ springeropen.com 